\title{
Trauma Exposure and Depression among Frontline Health Professionals During COVID-19 Outbreak in China: The Role of Intrusive Rumination and Organizational Silence
}

\section{Chaofan Li}

Centre for Health Management and Policy Research, School of Public Health, Cheeloo College of Medicine, Shandong University

Qiaobing Wu

Department of Applied Social Sciences, The Hong Kong Polytechnic University

\section{Debin Gu}

Institute for Hospital Management, Tsinghua University, Shenzhen

Shiguang Ni ( $\nabla$ ni.shiguang@sz.tsinghua.edu.cn )

Shenzhen International Graduate School, Tsinghua University

\section{Research Article}

Keywords: Trauma exposure, depression, intrusive rumination, organization silence, healthcare professional, COVID-19

Posted Date: September 16th, 2021

DOl: https://doi.org/10.21203/rs.3.rs-757274/v1

License: (c) (i) This work is licensed under a Creative Commons Attribution 4.0 International License.

Read Full License 


\section{Abstract}

Background: Healthcare professionals are a population exposed to especially high riskand stress during the COVID-19 outbreak. Several studies have demonstrated that healthcare professionals exposed to COVID-19 reported various affective disorders such as depressive symptoms, anxiety, insomnia, and distress. However, the mechanism underlying the association between trauma exposure and depressive symptom among frontline hospital staff has yet to be investigated. This study aims to assess the prevalence of depressive symptoms among frontline healthcare professionals in Shenzhen, China, particularly examining its association with trauma exposure, intrusive rumination and organizational silence.

Methods: Data of the study came from a time-lagged panel questionnaire survey with three waves of measurement from February, 2020 to May, 2020 at an infectious diseases hospital of Shenzhen which accommodated all the confirmed cases of COVID-19 patients. Using clustersampling design, a total of 134 frontline healthcare professionals directly involved inproviding diagnosis, treatment and nursing services for COVID-19 patients completed three wave web survey. A moderated mediation model was performed to examine the complex interplay among the major study variables.

Results: Trauma exposure was significantly related with depression of frontline healthcare professionals. Intrusive rumination mediated the effect of trauma exposure on depressive symptom, and organization silence moderated the relationship between intrusive rumination and depressive symptoms. Intrusive rumination showed stronger effect on depressive while organization silences was at a lower level.

Conclusions: This research demonstrates the pivotal role that intrusive rumination and organizational silence play in predicting the depressive symptoms among the frontline healthcare professionals coping with COVID-19.

\section{Introduction}

\subsection{Background}

The unprecedented COVID-19 pandemic has posed huge threats to the health and mental health of the humankind worldwide. Healthcare professionals (HCP) constitute one population at extremely high risk of both physical problems and mental issues who have to work for long hours in high-pressure environments and expose to various types of trauma [1,2]. Studies from China, Italy and United States have suggested that high proportion of healthcare professionals exposed to COVID-19 related working environments reported mental illness, such as depression, anxiety, insomnia, and distress [3-6]. Moreover, compared to healthcare professionals in other positions, frontline hospital staffs had higher likelihood of reporting depressive symptoms [7]. Several studies also discussed potential interventions, such as adaptive training, psychological support and assistance form psychologist, online psychological counseling [8], that could be taken to deal with the mental health issues of medical staff. However, evidence on explanatory mechanisms that linked depression symptom and trauma exposure among the 
frontline hospital staffs was relatively scarce. It calls for further research on explaining when and how frontline hospital staff experience depressive symptom, which is crucial to inform targeted and effective psychological interventions or support [9]. To address this research gap, this study aims to examine the magnitude of depressive symptoms among frontline hospital staffs in Shenzhen, the metropolis and border city of China's mainland and Hong Kong, during the outbreak of COVID-19, and to examine the potential mediators and/or moderators that explained the relationship between trauma exposure and depressive symptoms. Understanding the mechanism of how trauma exposure predicts depression is expected to have important theoretical, practical and policy implications.

\subsection{Theory and hypotheses}

\subsubsection{Trauma exposure and depression}

Depression is a pervasive negative mental health outcome of trauma exposure [10]. During the past decades, a series of epidemiological studies have reported that depression usually occur after trauma exposure [11-14]. According to a recent systematic review, the prevalence rate of depression among children and adolescents exposed to trauma was $24.2 \%, 2.6$ times higher than those unexposed or less exposed [15]. Specially, previous research has demonstrated that healthcare workers reported higher levels of depression during emerging infectious disease outbreak. As McAlonan and colleagues (2007) reported, during the SARS outbreak in 2003, frontline healthcare workers in acute care hospitals of Hong Kong not only demonstrated high chronic psychological stress but also depressive symptom and anxiety [16]. Liu also reported that hospital staff in Beijing exposed to SARS epidemic were at higher risk for depression [17]. As Lancee pointed out, working in a hospital treating SARS patients could represent a psychological trauma for healthcare workers [18]. The frontline hospital staff had to: 1 ) work for long hours and bear heavy workload and stress; 2) bear higher risk of being infected; 3 ) face the fear of bringing back the virus to household member thus trying to stay away from family [19]; and 4) accept their incapability to cure confirmed patients $[20,21]$. The emergency situation could constitute heavy psychological burden and lead to mental illness for frontline healthcare professionals. As such, we hypothesized that:

Hypothesis 1: Trauma exposure is positively related with depression of frontline healthcare professionals during the COVID-19 pandemic.

\subsubsection{Mediating role of intrusive rumination}

Rumination is usually defined as the process or tendency to think repetitively, persistently and uncontrollably on one's feelings and problems $[22,23]$. Previous studies have illustrated that rumination is associated with the development and maintenance of depression [24]. Rumination has been considered as an important construct to explain and predict depression from both theoretical and empirical aspect $[23,25]$. It should be noted that rumination is not a unitary construct but there are several subtypes $[24,26]$. It is important to distinguish the subtypes of rumination and corresponding effects on depression. A meta-analysis conducted by Olatunji reported that there was robust association 
between brooding rumination and depression, while the relations between other subtypes of rumination and depression appeared to be weak [25].

Moreover, there are several studies on the role of rumination in the relations between trauma exposure and depressive symptom. Nolen reported that the ruminative response styles on earthquake trauma exposure could lead to long periods of depression [27]. Cann delineated the posttraumatic cognitive process using rumination conceptual framework, in which rumination was categorized into two subtypes: intrusive rumination and deliberate rumination [28]. Intrusive rumination was defined as repetitive and uncontrollable thoughts focused on negative emotions. Ehring reported that intrusive rumination was an important factor contributing to depression after trauma exposure [29]. Roley also found that intrusive rumination moderated the relationship between posttraumatic stress disorder and major depression [30]. The response styles theory (RST) proposed by Nolen-Hoeksema (1991) explained the relationship between intrusive rumination and depressive symptom [31]. According to RST, intrusive rumination could exacerbate depression through several cognitive mechanism: 1) intrusive rumination enhanced individuals' frequency of recalling, understanding and interpreting his/her trauma exposure circumstances using negative thoughts; 2 ) intrusive rumination interfered with effective problem solving actions and instrumental behaviors by thinking in pessimistic and fatalistic ways, which aggravated the stressful circumstances; 3 ) intrusive rumination led to the loss of social support, which in turn fueled the magnitude and prolonged the duration of depressive symptom [23, 27].

According to what the empirical research and response styles theory implied, we hypothesized that:

Hypothesis 2: Invasive rumination will medicate the effect of trauma exposure on the depressive symptoms of frontline healthcare professionals during the COVID-19 pandemic.

1.2.3 Moderating role of organizational silence in the relationship between trauma exposure and depressive symptom

Organizational silence refers to the behaviors of employees intentionally withholding important opinions, information or ideas on work-related circumstances or problems [32,33]. According to the differences in employees' primary motivation, Vane Dyne categorized organizational silence into three specific subtypes: acquiescent, defensive and prosocial silence [33]. Previous studies reported that organizational silence in public organizations had serious negative consequences, such as creating heavy stress and mistrust, lowering ability to detect errors and misconduct, resulting in disengagement, and preventing communications and knowledge sharing [34, 35]. Specially, in hospital setting, the hierarchical organization structure, divisions between managers and healthcare professionals, and lack of cooperation and communication across teams may reinforce hospital organizational silence [36]. Moreover, there is evidence that organizational silence in healthcare settings during COVID-19 pandemic is serious [37]. There were two reasons why organizational silence among frontline hospital staff became a pressing issue. First, breaking down organizational silence and speaking up was very important for patient safety [38]. In the atmosphere of organizational silence, the perception of psychological safety of HCP decreased, and communication and discussion about practices and skills among HCPs also 
decreased $[39,40]$. Subsequently, the negative patient outcomes increased and healthcare quality was threated [41]. Second, organizational silence in workplace had impacts on both their personal mental health and organizational performance [32]. In terms of personal mental health, psychologists reported that inhibiting expression of emotion and thoughts might influence mental health and psychological functioning [42]. Specially, Morrison proposed that organizational silence could lead to high stress and dissatisfaction [37]. Furthermore, Knoll and colleagues found that there existed a negative association between four subtypes of organizational silence and employee burnout, which implied the potential associations between organizational silence and employee mental health and well-being over time [43].

As such, we developed the third hypothesis as the following:

Hypothesis 3: Organizational silence will moderate the associations between trauma exposure and depression through intrusive rumination. Specially, the mediation effects of intrusive rumination will be weaker when organizational silence is high.

The overall conceptual framework of the study demonstrating the mediation role of intrusive rumination in the relationships between trauma exposure and depression and the moderation effect of organizational silence is presented in figure 1 :

\section{Methods}

\subsection{Study population}

Data of this study were obtained from a time-lagged panel survey with three waves of measurement from February 2020 to May 2020. Informed consent was obtained from all the participants. The survey was conducted at an infectious diseases hospital of Shenzhen (Hospital T). This hospital accommodated all the 471 confirmed COVID-19 cases of Shenzhen during the outbreak and epidemic of COVID-19. The study sample was recruited through a cluster sampling strategy, targeting healthcare professionals who were directly involved in providing diagnosis, treatment and nursing care for COVID-19 patients in this hospital. All frontline healthcare professionals working with COVID-19 were asked to participate in this survey. The Wave 1 data was collected in February 2020, with a total of 492 healthcare professionals completing the survey. Among these, 241 participants were excluded from the data given high proportion of missing values, leaving 251 eligible participants for wave 2 and wave 3 survey. There were 241 and 117 participants excluded for nonresponse at wave 2 and wave 3, respectively. Therefore, in the final study sample, 134 respondents were followed for all three waves of surveys, with a $27.24 \%$ follow-up response rate.

\subsection{Measurement}

\subsubsection{Depressive symptoms}

Depressive symptoms were assessed at the 3rd wave survey, using the 12-items General Health Questionnaire (GHQ-12). Participating healthcare professionals were asked to rate on a 5-point Likert 
scale ranging from 1 (never) to 5 (always) on items like how often she/he felt "constantly under strain", "unhappy and depressed", etc. GHQ-12 has been reported as a valid tool for detecting depression in both general population and hospital staff. In the study, items 2, 5, 6, 9, 10 and 11 were scored directly, while items $1,3,4,7,8$ and 12 were reverse coded to ensure the direction of each item score was consistent. The average value of all the items was calculated to obtain an overall scale score. The Cronbach's a of the scale in this study was $0.88,0.85$, and 0.88 .

\subsubsection{Trauma exposure}

At the first wave of survey, we investigated the trauma exposure using a six-item questionnaire, the Explosion Exposure Questionnaire [44], which represented two dimensions of trauma exposure: direct exposure and damage. The direct exposure dimension consisted of three items: a) is there any relative confirmed to be a case or died during the COVID-19 pandemic? $(0$, no; 1 , infected; 2 , dead $)$; $b)$ is there any acquaintance confirmed to be a case or died during the $\operatorname{COVID-19}$ pandemic? $(0$, no; 1 , infected; 2 , dead); c) Whether you have witnessed someone infected or dead during the COVID-19 pandemic? $(0, n o ; 1$, yes).The damage dimension was measured by the following three items: $d$ ) What was the impact of COVID-19 pandemic on your health status? ( 0 , none; 1 , mild; 2 , medium; 3 , severe); e) What was the impact of COVID-19 pandemic on your property? ( 0 , none; 1 , mild; 2 , medium; 3 , severe); $f)$ What was the impact of COVID-19 pandemic on yourself? ( 0 , none; 1 , mild; 2 , medium; 3 , severe). The average score of the six items was calculated to form the overall scale score. The Cronbach's a of the Explosion Exposure Questionnaire in this study was 0.60 .

\subsubsection{Intrusive rumination}

At the second wave of survey, we assessed the intrusive rumination and organizational silence. Intrusive rumination was measured by three items from the simplified Chinese Version of Event Related Rumination Inventory (C-ERRI) [45]. C-ERRI was translated into Chinese by Dong and Liu from its English version developed by Cann [28]. As Dong and Cann reported, C-ERRI had high reliability and validity for measuring intrusive rumination $[28,45]$. The original inventory used ten items to assess intrusive rumination. In this study, to save responding time of frontline hospital staff, three key items out of the ten were selected from C-ERRI to form a short version: a) thoughts about the event distracted me or kept me from being able to concentrate; $b$ ) other things kept leading me to think about my experience; $c$ ) I tried not to think about the event, but could not keep the thoughts from my mind. Respondents were asked to rate on a 5-point Likert scale ranging from 1 (Strongly disagree) to 5 (Strongly agree). Mean value of the three items was considered as score of intrusive rumination. The Cronbach's a of the short version scale in the study was 0.89 .

\subsubsection{Organizational silence}

Organizational silence was assessed by the 6-item Employee Silence Scale (ESS) [46]. It consists of three construct dimensions: acquiescent silence, defensive silence and disregardful silence. Acquiescent silence means that employees keep silent and passively obey orders when they perceive they are not able 
to change the current situation. Two items measured acquiescent silence: a) The leaders have made decisions, and my opinion would not take any effect; $b$ ) The leaders would not change decisions, and there is no significance even I express my opinion. Defensive silence refers to employees not expressing their opinions in order to avoid conflicts and estrangement. It was measured by two items: c) Remain silence and restraint so as not to be the targets; d) It is not necessary to offend the leaders and colleagues to express my opinions. Disregardful silence refers to employees not giving any views or opinions for they have low organizational commitment in the present job or institution. Disregardful silence was assessed by two items: e) I am not concerned of the hospital affairs; f) I choose the "middle way", and will not say anything and bear any more responsibility. Participants were asked to rate six items on a 5-point Likert scale ranging from 1 (never) to 5 (always). Mean value of the six items was used as score of organizational silence. The Cronbach's a of this scale was 0.90 .

\subsubsection{Control variables}

Two factors were included as control variables in data analysis given their potential effects on depressive symptoms. First, gender was controlled as a dichotomous variable ( 0 , male; 1 , female). As reported, there was gender differences in the prevalence of depression among frontline healthcare professionals [5]. Second, it was also documented that years of working was associated with depressive symptoms and organizational silence among doctors and nurses $[47,48]$. Therefore, we included working years in the analysis model, which was categorized into four groups: $<=5$ years, $6-15$ years, $16-25$ years, and 26 years + .

\subsection{Procedure}

The survey was conducted online during the pandemic of COVID-19. The ethical approval of the survey was obtained from the Institutional Review Board of the Department of Psychology, Tsinghua University. Healthcare Professionals All healthcare professionals from that department were invited to complete the online survey. Once they agreed to participate, a WeChat link of Questionnaire Star was sent to them, with informed consents being attached to the survey Upon receipt of the completed questionnaires,, data could be obtained and downloaded from the sever of Questionnaire Star. The first wave of survey was conducted in February 2020, followed by the second and third wave of survey in March 2020 and May 2020, respectively.

\subsection{Data analysis}

The hypothesized second stage moderation model was tested using PROCESS v2.15 for SPSS provided by Hayes [49]. All continuous variables were mean centered prior to analysis, whereas gender was binary variable ( 0 , male; 1 , female) and working years was categorized into 4 groups $(1,<=5$ years; $2,6-15$ years; $3,16-25$ years and 4,26 years + ). Two steps were taken to test the hypothesized model: First, an ordinary least squares regression was performed to test the effects of trauma exposure on depressive symptoms; second, a moderated mediation model was tested with intrusive rumination as the mediator and organizational silence as the moderator. To test the significance of direct and indirect effect, 5000 
bootstrap samples were used to calculate the bounds of $95 \%$ confidence intervals. Last, we plot the interactions at three values of organizational silence (mean and $\pm 1 S D$ ) to visualize the conditional effects of the moderator.

\section{Results}

\subsection{Descriptive statistics and correlations analysis}

Table 1 shows the descriptive statistics of all independent and outcome variables and the Person's correlation. The negative correlation $(-0.18,-0.18$ and $-0.21, P<0.05)$ between gender and depression, trauma exposure and intrusive rumination demonstrated that the male participants reported higher levels of depressive symptoms. Moreover, there were significant positive correlations between working years and intrusive rumination $(0.31, P<0.01)$ and organizational silence $(0.17, P<0.05)$. Trauma exposure, intrusive rumination and organizational silence were all significantly and positively correlated with depressive symptoms. The correlation coefficients were $0.21(P<0.05), 0.54(P<0.01)$ and $0.37(P<0.01)$ respectively. Furthermore, trauma exposure, intrusive rumination and organizational silence were positively associated with each other.

Table 1. Descriptive statistics and pairwise correlations among study variables

\begin{tabular}{|lllllllll|}
\hline & Mean & $S d$ & 1 & 2 & 3 & 4 & 5 & 6 \\
\hline Gender & 0.70 & 0.46 & 1.00 & & & & & \\
\hline Working years & 2.09 & 0.90 & -0.10 & 1.00 & & & & \\
\hline Depression & 2.34 & 0.62 & $-0.18^{*}$ & 0.06 & 1.00 & & & \\
\hline Trauma exposure & 2.07 & 0.44 & $-0.18^{*}$ & 0.08 & $0.21^{*}$ & 1.00 & & \\
\hline Intrusive rumination & 2.42 & 1.01 & $-0.21^{*}$ & $0.31^{\star \star}$ & $0.54^{\star \star}$ & $0.33^{\star \star}$ & 1.00 & \\
\hline Organizational silence & 2.77 & 0.89 & -0.08 & $0.17^{\star}$ & $0.37^{\star \star}$ & $0.22^{*}$ & $0.48^{\star \star}$ & 1.00 \\
\hline
\end{tabular}

Note: $N=134 .{ }^{*}, P<0.05 ;{ }^{* *}, P<0.01$.

\subsection{Mediation and moderation analysis}

As shown in Step 1, Table 2, trauma exposure was significantly positively related with depression of healthcare professionals $(\beta=0.18, P<0.05)$, adjusting for gender and working years. Results at step 2 showed that trauma exposure was positively correlated with intrusive rumination $(\beta=0.66, P<0.01)$. Furthermore, intrusive rumination was also positively correlated with depressive symptoms $(\beta=0.31$, $P<0.01)$. This supported our second hypothesis that intrusive rumination served as a mediator in the relationship between trauma exposure and depression. 
Moreover, organizational silence moderated the association between intrusive rumination and depression. The coefficients of interaction terms of intrusive rumination and organizational silence was $-0.11(P<0.05)$, indicating that organizational silence attenuated the positive correlations between intrusive rumination and depression.

Table 2 Results of the Moderated Mediation

\begin{tabular}{|c|c|c|c|c|c|c|}
\hline & \multicolumn{3}{|l|}{ Step 1} & \multicolumn{3}{|c|}{ Step 2} \\
\hline & $\beta$ & $S E$ & $T$ & $\beta$ & $S E$ & $t$ \\
\hline \multicolumn{7}{|l|}{ Mediator: Intrusive rumination } \\
\hline Gender & & & & -0.29 & 0.17 & -1.65 \\
\hline Working year & & & & 0.30 & 0.09 & $3.41^{\star *}$ \\
\hline Trauma exposure & & & & 0.66 & 0.18 & $3.64^{* *}$ \\
\hline$R^{2}$ & & & & & & $0.21^{\star *}$ \\
\hline \multicolumn{7}{|l|}{ Dependent variable: Depression } \\
\hline Gender & -0.14 & 0.12 & -1.67 & -0.12 & 0.10 & -1.19 \\
\hline Working years & 0.03 & 0.06 & 0.39 & -0.07 & 0.05 & -1.30 \\
\hline Trauma exposure & $0.18^{\star}$ & 0.12 & 2.13 & 0.02 & 0.11 & 0.16 \\
\hline Intrusive rumination & & & & 0.31 & 0.05 & $5.67^{* *}$ \\
\hline Organizational silence & & & & 0.10 & 0.06 & 1.78 \\
\hline Intrusive rumination*Organization & silence & & & -0.11 & 0.04 & $-2.47^{*}$ \\
\hline$R^{2}$ & & & $0.26^{*}$ & & & $0.36^{* *}$ \\
\hline$R^{2}$ & & & & & & 0.11 \\
\hline
\end{tabular}

Note: $N=134 .{ }^{*}, P<0.05 ;{ }^{* *}, P<0.01$.

Figure 2 shows that intrusive rumination was significantly related with depression of hospital staffs while organizational silence was at either low $(\beta=0.40, P<0.01)$, medium $(\beta=0.29, P<0.01)$ or high $(\beta=0.19, P<0.05)$ level. However, intrusive rumination demonstrated stronger effects on depressive symptoms when organizational silence as at lower level, indicating a significant moderation effect of organizational silence on the relationship between intrusive rumination and depression of frontline healthcare professionals. 
The bootstrap confidence intervals of conditional indirect effect analysis shown in Table 3 were all positive and did not include zero. This implied that intrusive rumination mediated the association between trauma exposure and depression regardless of the level of organizational silence. Specially, the mediation effects of intrusive rumination appeared to be stronger when organizational silence was at a lower level

Table 3 Conditional Indirect Effect of Intrusive Rumination on Depression at Different Levels of Organizational Silence

\begin{tabular}{|lllll|}
\hline Mediator & Moderator: Organizational silence & Effect & SE & Bootstrap Cl \\
\hline Invasive rumination & Low & 0.27 & 0.10 & {$[0.11 ; 0.50]$} \\
\hline Invasive rumination & Medium & 0.20 & 0.07 & {$[0.08 ; 0.38]$} \\
\hline Invasive rumination & High & 0.14 & 0.07 & {$[0.04 ; 0.31]$} \\
\hline
\end{tabular}

\section{Discussion}

\subsection{Correlations between trauma exposure and depressive symptoms}

Consistent with our hypothesis 1 , the study results suggested that trauma exposure was positively correlated with depression of hospital staffs. This indicated that healthcare professionals at higher risk of exposure to coping with COVID-19 affairs were more likely to suffer from depression. This finding was supported by other cross-sectional studies [4, 21] and systematic review [5] as well, which all reached similar conclusions that depression and other mental health problems were highly prevalent among the frontline healthcare professionals with exposure to COVID-19 risks or environments. They not only had heave physiological stress to provide diagnosis, treatment and nursing care, but also bear high levels of psychological distress, such as fearing infection or death of family members and friends, worrying about serious damage of COVID-19 on their own safety, health, family's property and career development. This implied that trauma exposure to long-term physiological and psychological stress are prominent risk factors for depression among healthcare professionals exposed to treating patients with COVID-19.

\subsection{The mediation effects of intrusive rumination}

In line with our hypothesis 2 , it was revealed that intrusive rumination fully medicated the effect of trauma exposure on depressive symptoms of frontline healthcare professionals. This demonstrated that intrusive rumination, as a cognitive mechanism, played an important role in the process that trauma exposure influenced depressive symptoms. Previous studies have found that intrusive rumination commonly occurred after long-term trauma exposure [29, 50-53]. Furthermore, previous study argued that intrusive rumination prolonged the period and exacerbated the degree of the negative effects of trauma exposure [25]. Therefore, it was understandable that individuals exposed to trauma events who had higher level of intrusive rumination would have more severe depressive symptoms. Consistent with these 
findings, previous empirical research has also proved that intrusive rumination could mediate the effects of negative affect, such as uncertainty, emotion, and stress, on depressive symptoms [54-58].

\subsection{The moderation effect of organizational silence}

In accordance with hypothesis 3 , the moderated mediation model results in this study suggested that organizational silence moderated the relationship between intrusive rumination and depressive symptoms. Similar to previous research findings, this study demonstrated that organizational silence was not only positively related with depression [59-61], but also intrusive rumination. Employees who could not express their ideas and feelings may potentially suffer from high psychological distress, depression and other mental health problems [37]. Specially, the findings demonstrated that the relationship between intrusive rumination and depression varied by levels of organizational silence. That is, at high level of organizational silence, intrusive rumination would show greater effects on depressive symptoms. Thus, if hospital healthcare professionals shared less of their ideas and opinions, intrusive rumination would reinforce their experience of trauma exposure thus increasing the magnitude of depressive symptoms.

\subsection{Limitations and future research}

The results this research should be interpreted with caution due to several limitations. First, the selfreported measures may lead to response bias and common-method bias. Future research should try to use triangulation to collect multi-resource data and use multi-method assessments to measure each psychological concept. Second, the time-lagged study design could examine the association between trauma exposure and depression. However, we cannot estimate the causal effects of trauma exposure on depression. Future research could use natural experiment study design or longitudinal study design with repeated measured to test the effects of targeted psychological support, such as decreasing intrusive rumination and organizational silence, on the prevalence of depressive symptoms. Last, participants of this study were concentrated within one hospital, which may influence the representativeness of the sample and the generalizability of the conclusion. Future study could try to recruit a more representative municipal, provincial or national sample to test the hypothesized model.

\subsection{Contributions}

Despite these limitations, this study expects to contribute to the literature in both theoretical and practical terms. First, the findings help explain whether and how trauma exposure predicts depressive symptoms. With consideration of the mediation role of intrusive rumination and moderating effect of organizational silence, this study will advance the use of response style theory and cognitive appraisal theory in interpreting depressive symptoms among a unique population. Second, understanding the explanatory mechanism among trauma exposure, intrusive rumination, organizational silence and depression also have important implications for designing targeted and appropriate interventions to decrease depressive symptoms among frontline healthcare professionals.

\section{Conclusion}


In sum, this research demonstrates the pivotal role that intrusive rumination and organizational silence plays in explaining the depressive symptoms among the frontline healthcare professionals working in the designated hospital and coping with COVID-19. We expect these research findings to be informative for psychologists and policy-makers to develop specific interventions or create a more comfortable working environment that provide hospital staff with psychological support and alleviate their mental health burden.

\section{Declarations}

\section{Acknowledgements}

The authors gratefully acknowledge the research members, families, and frontline health professionals who took part in this research, without whom it would not have been possible.

\section{Authors' contributions}

CL: Conceptualization, Formal analysis, Methodology, Software, Validation, Visualization, Writing original draft, Writing - review \& editing.

QW: Conceived and designed the study, Writing - review \& editing.

DG: Writing - review \& editing.

SN: Conceived and designed the study, Data curation, Funding acquisition, Investigation, Methodology, Project administration, Resources.

\section{Funding}

This study was funded by the Humanities and Social Sciences Foundation of China Ministry of Education (18YJAZH065), Shenzhen Key Research Base of Humanities and Social Sciences, the Interdisciplinary Research Project of Graduate School of Shenzhen of Tsinghua University (JC2017005) and the Guangdong Natural Science Foundation (Grant No. 2020A1515010949).

\section{Availability of data and materials}

The datasets used and/or analyzed during the current study are available from the corresponding author on reasonable request.

\section{Ethics approval and consent to participate}

This study was approved through the Institutional Review Board of the Department of Psychology (Ref: 202004), Tsinghua University and research meets the ethical guidelines, including adherence to the legal requirements of the study country. All the participants provided written informed consent. All procedures 
contributing to this work comply with the ethical standards of the relevant national and institutional committees on human experimentation and with the Helsinki Declaration of 1975, as revised in 2008.

Consent for publication

Not applicable.

Competing interests

The sponsors were not involved in study design; in the collection, analysis and interpretation of data; in the writing of the report; and in the decision to submit the article for publication.

The author(s) declared no potential conflicts of interest with respect to the research, authorship, and/or publication of this article.

\section{References}

1. Braquehais MD, Vargas-Caceres S, Gomez-Duran E, Nieva G, Valero S, Casas M, Bruguera E: The impact of the COVID-19 pandemic on the mental health of healthcare professionals. QJM 2020, 113(9):613-617.

2. Greenberg N: Mental health of health-care workers in the COVID-19 era. Nat Rev Nephro/ 2020, 16(8):425-426.

3. Du J, Dong L, Wang T, Yuan C, Fu R, Zhang L, Liu B, Zhang M, Yin Y, Qin J et al: Psychological symptoms among frontline healthcare workers during COVID-19 outbreak in Wuhan. Gen Hosp Psychiatry 2020, 67:144-145.

4. Lai J, Ma S, Wang Y, Cai Z, Hu J, Wei N, Wu J, Du H, Chen T, Li R et al: Factors Associated With Mental Health Outcomes Among Health Care Workers Exposed to Coronavirus Disease 2019. JAMA Netw Open 2020, 3(3):e203976.

5. Pappa S, Ntella V, Giannakas T, Giannakoulis VG, Papoutsi E, Katsaounou P: Prevalence of depression, anxiety, and insomnia among healthcare workers during the COVID-19 pandemic: A systematic review and meta-analysis. Brain Behav Immun 2020, 88:901-907.

6. Pearman A, Hughes ML, Smith EL, Neupert SD: Mental Health Challenges of United States Healthcare Professionals During COVID-19. Front Psychol 2020, 11:2065.

7. Lin K, Yang BX, Luo D, Liu Q, Ma S, Huang R, Lu W, Majeed A, Lee Y, Lui LMW et al: The Mental Health Effects of COVID-19 on Health Care Providers in China. Am J Psychiatry 2020, 177(7):635-636.

8. Tomlin J, Dalgleish-Warburton B, Lamph G: Psychosocial Support for Healthcare Workers During the COVID-19 Pandemic. Front Psychol 2020, 11:1960.

9. Holmes EA, O'Connor RC, Perry VH, Tracey I, Wessely S, Arseneault L, Ballard C, Christensen H, Cohen Silver R, Everall I et al: Multidisciplinary research priorities for the COVID-19 pandemic: a call for action for mental health science. The Lancet Psychiatry 2020, 7(6):547-560. 
10. Wozniak JD: Trauma Exposure Depressive Symptoms and Responding to Positive Positive Events and Affect in Young Adults. Seattle: Seattle Pacific University; 2020.

11. Goldstein LA, Dinh J, Donalson R, Hebenstreit CL, Maguen S: Impact of military trauma exposures on posttraumatic stress and depression in female veterans. Psychiatry Res 2017, 249:281-285.

12. Qi J, Yang X, Tan R, Wu X, Zhou X: Prevalence and predictors of posttraumatic stress disorder and depression among adolescents over 1 year after the Jiuzhaigou earthquake. J Affect Disord 2020, 261:1-8.

13. Ying LH, Wu XC, Lin CD, Chen C: Prevalence and predictors of posttraumatic stress disorder and depressive symptoms among child survivors 1 year following the Wenchuan earthquake in China. Eur Child Adolesc Psychiatry 2013, 22(9):567-575.

14. Zhou X, Wu X, An Y: Understanding the Relationship between Trauma Exposure and Depression among Adolescents after Earthquake: The Roles of Fear and Resilience. Front Psychol 2016, 7:2044.

15. Vibhakar V, Allen LR, Gee B, Meiser-Stedman R: A systematic review and meta-analysis on the prevalence of depression in children and adolescents after exposure to trauma. J Affect Disord 2019, 255:77-89.

16. McAlonan GM, Lee AM, Cheung V, Cheung C, Tsang KW, Sham PC, Chua SE, Wong JG: Immediate and sustained psychological impact of an emerging infectious disease outbreak on health care workers. Can J Psychiatry 2007, 52(4):241-247.

17. Liu X, Kakade M, Fuller CJ, Fan B, Fang Y, Kong J, Guan Z, Wu P: Depression after exposure to stressful events: lessons learned from the severe acute respiratory syndrome epidemic. Compr Psychiatry 2012, 53(1):15-23.

18. Lancee WJ, Maunder RG, Goldbloom DS, Study S: Prevalence of psychiatric disorders among Toronto hospital workers one to two years after the SARS outbreak. Psychiat Serv 2008, 59(1):91-95.

19. Wilson W, Raj JP, Rao S, Ghiya M, Nedungalaparambil NM, Mundra H, Mathew R: Prevalence and Predictors of Stress, anxiety, and Depression among Healthcare Workers Managing COVID-19 Pandemic in India: A Nationwide Observational Study. Indian J Psychol Med 2020, 42(4):353-358.

20. Guo J, Liao L, Wang B, Li X, Gu Y: Psychological effects of COVID-19 on hospital staff: A national cross-sectional survey in mainland China. Vasc Investig Ther 2021, 4(1):6-11.

21. Lu W, Wang H, Lin Y, Li L: Psychological status of medical workforce during the COVID-19 pandemic: A cross-sectional study. Psychiatry Res 2020, 288:112936.

22. Brinker JK, Dozois DJA: Ruminative thought style and depressed mood. Journal of Clinical Psychology 2009, 65(1):1-19.

23. Nolen-Hoeksema S, Wisco BE, Lyubomirsky S: Rethinking Rumination. Perspect Psychol Sci 2008, 3(5):400-424.

24. Tanner A, Voon D, Hasking P, Martin G: Underlying Structure of Ruminative Thinking: Factor Analysis of the Ruminative Thought Style Questionnaire. Cognit Ther Res 2013, 37(3):633-646. 
25. Olatunji BO, Naragon-Gainey K, Wolitzky-Taylor KB: Specificity of Rumination in Anxiety and Depression: A Multimodal Meta-Analysis. Clinical Psychology Science \& Practice 2013, 20(3):225257.

26. Treynor W, Gonzalez R, Nolen-Hoeksema S: Rumination reconsidered: A psychometric analysis. Cognit Ther Res 2003, 27(3):247-259.

27. Nolen-Hoeksema S, Morrow J: A prospective study of depression and posttraumatic stress symptoms after a natural disaster: The 1989 Loma Prieta earthquake. Journal of Personality and Social Psychology 1991, 61(1):115-121.

28. Cann A, Calhoun LG, Tedeschi RG, Triplett KN, Vishnevsky T, Lindstrom CM: Assessing posttraumatic cognitive processes: the Event Related Rumination Inventory. Anxiety Stress Coping 2011, 24(2):137156.

29. Ehring T, Frank S, Ehlers A: The Role of Rumination and Reduced Concreteness in the Maintenance of Posttraumatic Stress Disorder and Depression Following Trauma. Cognit Ther Res 2007, 32(4):488506.

30. Roley ME, Claycomb MA, Contractor AA, Dranger P, Armour C, Elhai JD: The relationship between rumination, PTSD, and depression symptoms. J Affect Disord 2015, 180:116-121.

31. Nolen-Hoeksema S: Responses to Depression and Their Effects on the Duration of Depressive Episodes. Journal of Abnormal Psychology 1991, 100(4):569-582.

32. Donaghey J, Cullinane N, Dundon T, Wilkinson A: Reconceptualising employee silence. Work Employ Soc 2011, 25(1):51-67.

33. Van Dyne L, Ang S, Botero IC: Conceptualizing employee silence and employee voice as multidimensional constructs. J Manage Stud 2003, 40(6):1359-1392.

34. Gambarotto F, Cammozzo A: Dreams of silence: Employee voice and innovation in a public sector community of practice. Innovation 2014, 12(2):166-179.

35. Hassan S, DeHart-Davis L, Jiang Z: How empowering leadership reduces employee silence in public organizations. Public Administration 2019, 97(1):116-131.

36. Erkutlu $\mathrm{H}$, Chafra $\mathrm{J}$ : Leader's integrity and employee silence in healthcare organizations. Leadership in Health Services 2019, 32(3):419-434.

37. Morrison EW: Employee Voice and Silence. Annual Review of Organizational Psychology and Organizational Behavior 2014, 1(1):173-197.

38. Okuyama A, Wagner C, Bijnen B: Speaking up for patient safety by hospital-based health care professionals: a literature review. BMC Health Serv Res 2014, 14:61.

39. Doo EY, Choi S: Effects of horizontal violence among nurses on patient safety: Mediation of organisational communication satisfaction and moderated mediation of organisational silence. $J$ Nurs Manag 2021, 29(3):526-534.

40. Doo EY, Kim M: Effects of hospital nurses' internalized dominant values, organizational silence, horizontal violence, and organizational communication on patient safety. Res Nurs Health 2020, 
43(5):499-510.

41. Henriksen K, Dayton E: Organizational silence and hidden threats to patient safety. Health Serv Res 2006, 41 (4 Pt 2):1539-1554.

42. Gross JJ, Levenson RW: Hiding feelings: the acute effects of inhibiting negative and positive emotion. J Abnorm Psychol 1997, 106(1):95-103.

43. Knoll M, Hall RJ, Weigelt O: A longitudinal study of the relationships between four differentially motivated forms of employee silence and burnout. J Occup Health Psychol 2019, 24(5):572-589.

44. Guo W: Prevalence and predicting factors for negative emotions among adolescents exposed to an explosion. Medicine. Jinan: Shandong University; 2015.

45. Dong C, Gong S, Liu X: Reliability and validity of the simplified Chinese Version of Event Related Rumination Inventory among accidentally injured patients. Chin J Nurs 2013, 48(9):831-834.

46. Zheng X, Ke J, Shi J, Zheng X: Survey on Employee Silence and the Impact of Trust on it in China. Acta Psychologica Sinica 2008, 40(2):219-227.

47. Sun Z, Cui Y, Liu X, Yv X, Ren D, Chen Y: Prevelence of Depression Symptom and Influencing Factor among Doctors and Nurses. Chinese Mental Health Journal 1997, 11(5):317.

48. Zhang A, Zhang Y, Nan L, Gao Y: Analylsis of status quo and influencing factors of nurse organizational silence. Chinese Nursing Research 2018, 32(6):972-975.

49. Hayes AF: Introduction to Mediation, Moderation, and Conditional Process Analysis, second edn. New York: The Guilford Press; 2018.

50. Arnesen $\mathrm{CH}$, Bjørndal K, Vaaland K: Long-term Effects of Trauma Exposure on Inhibition and Rumination among Utøya Survivors. The University of Bergen; 2015.

51. Ehlers A, Steil R: Maintenance of intrusive memories in posttraumatic stress disorder: a cognitive approach. Behav Cogn Psychother 1995, 23(3):217-249.

52. Elwood LS, Hahn KS, Olatunji BO, Williams NL: Cognitive vulnerabilities to the development of PTSD: a review of four vulnerabilities and the proposal of an integrative vulnerability model. Clin Psychol Rev 2009, 29(1):87-100.

53. Viana AG, Paulus DJ, Garza M, Lemaire C, Bakhshaie J, Cardoso JB, Ochoa-Perez M, Valdivieso J, Zvolensky MJ: Rumination and PTSD symptoms among trauma-exposed Latinos in primary care: Is mindful attention helpful? Psychiatry Res 2017, 258:244-249.

54. Burwell RA, Shirk SR: Subtypes of rumination in adolescence: associations between brooding, reflection, depressive symptoms, and coping. J Clin Child Adolesc Psychol 2007, 36(1):56-65.

55. Meiser-Stedman R, Dalgleish T, Glucksman E, Yule W, Smith P: Maladaptive cognitive appraisals mediate the evolution of posttraumatic stress reactions: A 6-month follow-up of child and adolescent assault and motor vehicle accident survivors. J Abnorm Psychol 2009, 118(4):778-787.

56. Schoofs H, Hermans D, Raes F: Brooding and Reflection as Subtypes of Rumination: Evidence from Confirmatory Factor Analysis in Nonclinical Samples using the Dutch Ruminative Response Scale. $J$ Psychopathol Behav Assess 2010, 32(4):609-617. 
57. Verstraeten K, Bijttebier P, Vasey MW, Raes F: Specificity of worry and rumination in the development of anxiety and depressive symptoms in children. Br J Clin Psychol2011, 50(4):364-378.

58. Yook K, Kim KH, Suh SY, Lee KS: Intolerance of uncertainty, worry, and rumination in major depressive disorder and generalized anxiety disorder. J Anxiety Disord 2010, 24(6):623-628.

59. Bagheri G, Zarei R, Aeen MNJITom: Organizational silence (basic concepts and its development factors). Ideal Type of management 2012, 1(1):47-58.

60. Ebrahimi A, Bagheri Gara Bollagh H, Eynali M, Baki Hashemi MM: The Role of Organizational Trauma on Silence and Organizational Cynicism in a Health Service Provider Center. The Neuroscience Journal of Shefaye Khatam 2019, 7(3):51-60.

61. Nikmaram S, Yamchi HG, Shojaii S, Zahrani MA, Alvani SMJWASJ: Study on relationship between organizational silence and commitment in Iran. World App/ Sci J 2012, 17(10):1271-1277.

\section{Figures}

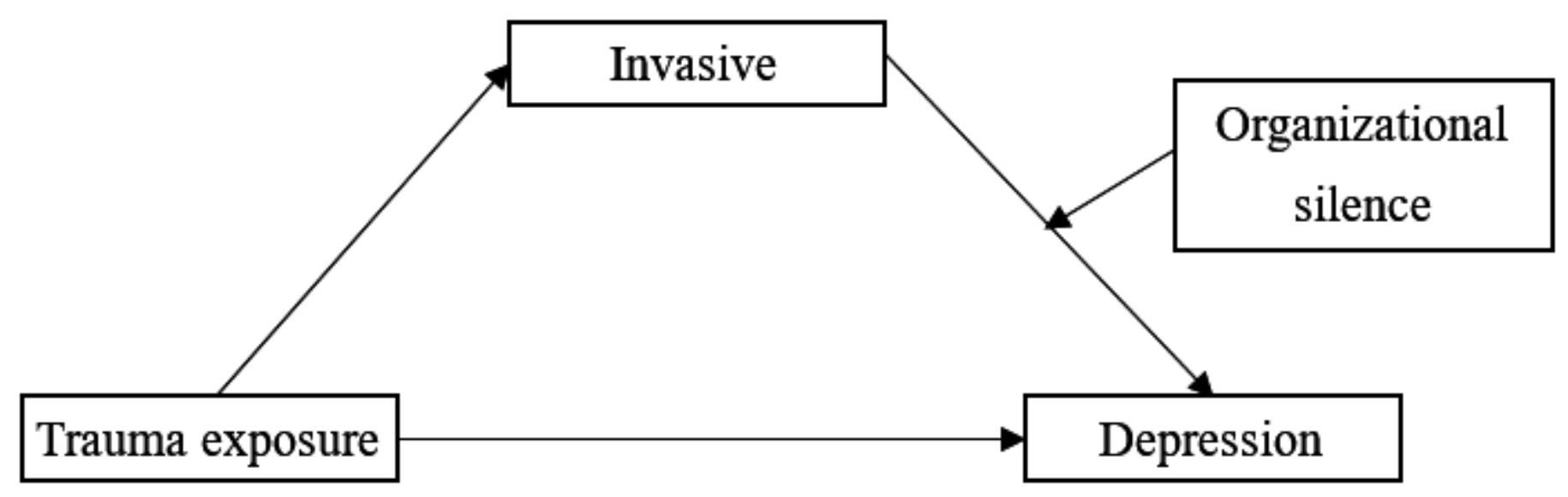

Figure 1

The hypothesized conceptual framework 


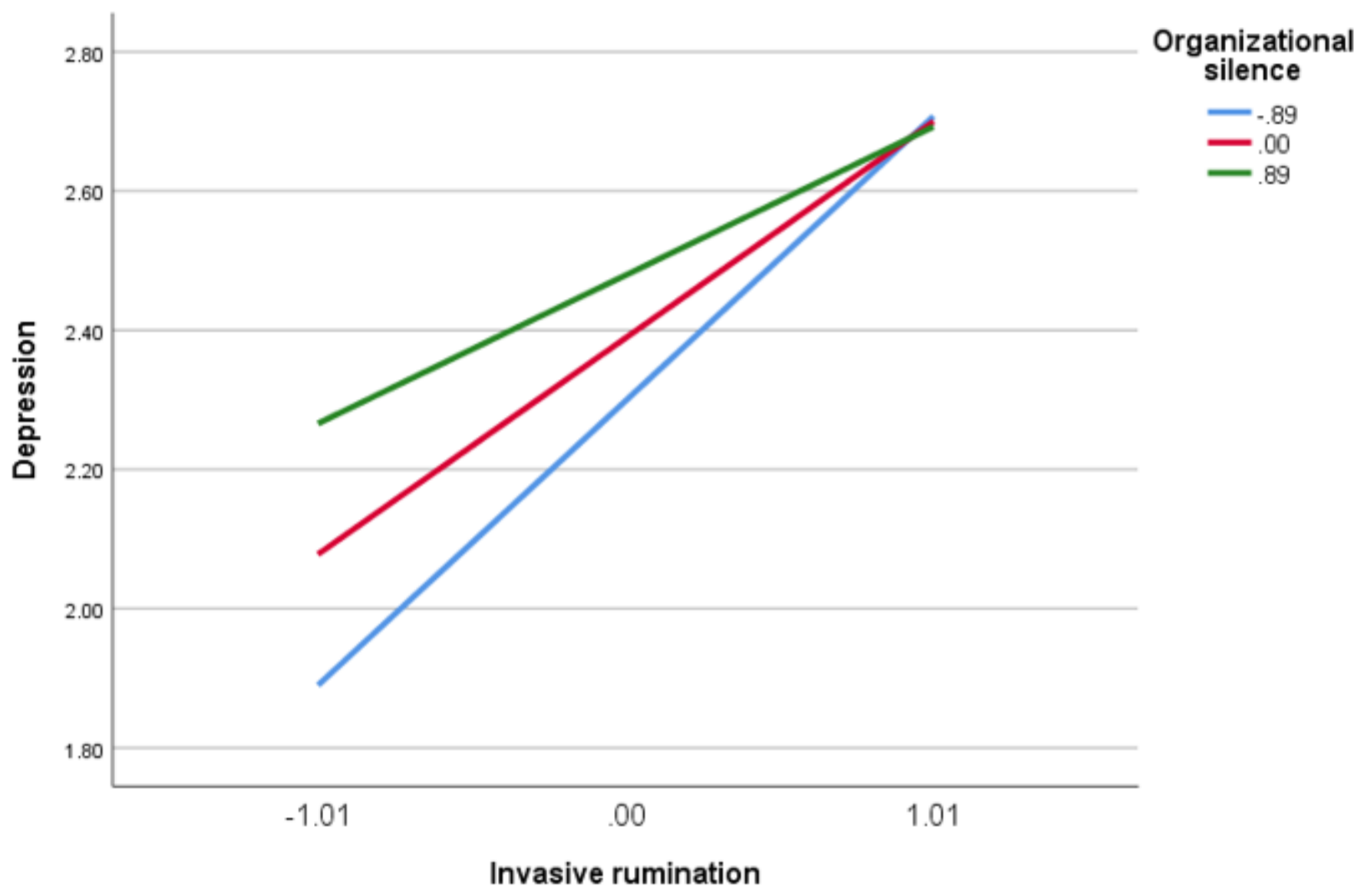

Figure 2

The interaction of Invasive Rumination and Organizational silence 